\title{
The Impact of Media Coverage and Emergency Strategies on the Rumor Spreading
}

\author{
Liang'an Huo $\mathbb{D}$ and Yingying Cheng \\ Business School, University of Shanghai for Science and Technology, Shanghai 200093, China \\ Correspondence should be addressed to Liang’an Huo; huohuolin@yeah.net
}

Received 6 September 2018; Accepted 5 November 2018; Published 9 December 2018

Academic Editor: Yukihiko Nakata

Copyright (c) 2018 Liang'an Huo and Yingying Cheng. This is an open access article distributed under the Creative Commons Attribution License, which permits unrestricted use, distribution, and reproduction in any medium, provided the original work is properly cited.

\begin{abstract}
On the basis of nonlinear dynamic model, we research the propagation of rumors after emergencies, describe the impact of media coverage and emergency strategies by government on the transmission dynamics of information, and then obtain the basic reproduction number of rumor spreading. In order to overcome the limit of traditional methods of the static decision problem, the dynamic optimal control model for the rumor spreading is proposed based on the theorem of the optimal control. An optimal objective based on the maximum social utility is established and the optimal solution is acquired by using the Pontryagin Maximum Principle. Finally, numerical simulations show that the dynamic optimal control method has obvious superiority in modeling compared with the method without control. By means of the dynamic optimal control of media coverage and emergency strategies for government, the final rumor scale and the peak of spreaders can be effectively reduced.
\end{abstract}

\section{Introduction}

When emergencies occur, people's thirst for information is different from normal. If the propagation of information is not transparent, detailed, and controlled by the government and the media at this time, it may lead to people's psychological and emotional tension, unnecessary public panic, and economic loss [1-4]. On the contrary, if there is a clear channel of information dissemination and effective information dissemination mode among the government, the media, and the public, this will be beneficial to reduce the social panic and prevent the expansion and dissemination of adverse effects in the event. At the same time, the information interactive platform can better establish the credibility of the government and the media.

In recent years, many scholars have discussed the rumor spreading from different perspectives through qualitative analysis and theoretical modeling. Rapoport [5] first uses the epidemic model to study the spread of information. Goffman and Newill [6] compare the similarities between the spread of disease and rumor and use the stability of the epidemic model to explain the final propagation state of the rumor.
Daley and Kendall [7] put forward a mathematical model of rumor spreading in 1960s. After that, scholars have expanded the classic D-K model. Based on summarizing the similarities and differences between the spread of infectious diseases and the mechanism of rumor diffusion, Klaus [8] emphatically expounds the reasonableness of using the epidemic model to explain the spread of rumors.

With the development of dynamic theories, rumor spreading based on dynamics has attracted more and more attention. Dickinson [9] compared the spreading of epidemic with the rumor spreading mechanism. And they considered the propagation is a time-varying process, which can be subsumed under a general class of transient processes whose time-dependent evolution can be characterized exactly. According to the characteristics of audience, Thompson [10] divide the crowd into two categories, active and indifferent, set up a dynamic model of rumor spreading, and analyze the strategy of controlling the spread of rumors. Based on this, a large number of scholars [11-14] have expanded the rumor propagation model from the perspective of activity.

Media reports are known as "subcutaneous injection" and "magic bullet" theory, and it is believed that media 
coverage has a great influence on public's understanding and emergency management [15]. In the outbreak of large-scale infectious diseases, the media coverage will affect the final spread of disease; moderate coverage can limit the spread of infectious diseases and reduce the loss of infectious disease transmission. However, overreporting can cause panic and also bring disastrous consequences out of control [16, 17]. Referring to the theory of disease, Zhao et al. [18] built a model verifying that the media with high ratings but low credibility will cause widespread panic and cause a lot of rumors. Jane M Heffernan and Shannon Collinson [19] have applied the "media function" that affects the transmission rate in the mathematical epidemiology model, which allowed the effects of mass media during disease outbreaks to be studied in mathematical modeling literature. Jean M. Tchuenche and Chris T. Bauch [20] established a susceptibleinfected-hospitalized-recovered model with vital dynamics, in which the media coverage of disease incidence and disease prevalence can influence people to reduce their contact rates. And the media function is merged into the model by using the exponential decreasing function skillfully. Luo et al. [21] explored the impact of positive news on rumor spreading in scale-free networks. It is concluded that most of the rumors related to hot events or emergencies can be propagated rapidly on the hotbed of online social networks. Huo et al. $[22,23]$ have made a great contribution in rumors spreading, and their results indicate that the media can be regarded as a good indicator in controlling the occurrence and spreading of rumor.

When an emergency occurs, it will cause or may cause serious social harm. In order to avoid losses, some measures must be taken as soon as possible in response to the sudden incidents, such as natural disasters, accident disasters, public health events, and social security incidents. However, the main effect of media coverage is to popularize science education, improve the identification of the ignorant, reduce the probability of the ignorant believing rumors, and thus reduce the spreading rate of rumor dissemination, rather than prevent the spread of rumors. On the contrary, when rumors break out, relevant emergency strategies can not only prevent individuals from believing in rumors, but also reduce the spreader's enthusiasm for dissemination. Huo et al. [24], based on utility theory, proposed a model to describe the interplay between rumor spreading and authorities' actions. Through numerical simulation, it is found that positive actions in the government can improve rumor management in emergencies and generate positive social effects. To study the spread of rumor in emergencies, Chen et al. [25] investigated a novel rumor spreading model with latent, constant recruitment, and varying total population. $\mathrm{Li}$ et al. [26] explored the impact of punishment of governments and sensitivity of individuals on the rumor spreading and then drew a conclusion that improving the punishment of government and increasing the sensitivity of individuals can effectively control the spreading of rumor.

The spread of rumor not only caused the people to panic, but also brought huge economic losses. Therefore, many scholars have made great contributions to the immune control of rumors $[27,28]$. However, controlling rumors is only the first goal. Saving control costs is equally important. Thus, optimal control theory has been widely applied in rumor governance in recent years. Based on the controlled heterogeneous node-based SIRS model, Huo et al. [29] considered an optimal control of rumor spreading model with psychological factors and time delay. Kandhway et al. [30] based on SIS and SIR models considered fixed and time varying information spreading rate and formulated two optimal control models, and then they proved the effectiveness of the optimal control strategy against the constant control strategy, a heuristic control strategy and no control. Huo et al. [31] established an optimal control model based on the SEIR model with latent period to study rumor management strategy. Inspired by the classical SIR model, Xu et al. [32] proposed a new rumor spreading model, which regards the transfer probability of nodes from susceptive to infected as a variable instead of a constant. Jeong et al. [33] proposed a rumor model with three control strategies for preventing the spread of rumor: (1) announcing the truth before ignorant receives rumor, (2) punishing spreaders, and (3) deleting information of the rumor in media, and considering optimal control problems to minimize the number of spreaders while minimizing the cost of three control strategies for preventing the spread of rumors.

From the previous researches, the scholars mainly focus on the state of the final spreading, but ignore the influence factors and the control problems during the process. The individual's spreading behavior will be determined by the amount of information obtained by an individual preference; meanwhile, the media and government measures will affect the rumor spreading. The facts indicate that the popularization of scientific knowledge, emergency strategies, authoritative information published by the media, expansion of information coverage, and guiding public opinion are the key to rumor governance in emergency management.

Considering the positive impact of media coverage and the emergency strategies of government, we extended the classical DK model. On the basis of the optimal control theory, the optimal control strategy of rumor spreading is discussed, and a feasible management and control scheme is proposed for the control of the rumor in emergency management and obtainment of an emergency management strategy with maximization of social utility.

The organization of this paper is as follows: the rumor spreading model is formulated in the next section. Section 2 describes an analysis of the equilibrium, giving existence conditions and a kind of threshold condition for rumors outbreaks. Section 3 analyzes the optimal control problem in detail. We present some numerical simulations to illustrate the established results in Section 4. Finally, Section 5 outlines the work and some topics that are worthy of study.

\section{The Mathematical Model with the Impact of Media Coverage and Emergency Strategies}

The spread of rumors will be influenced by media coverage. The media coverage improves personal discrimination ability through daily popular science information, thereby reducing the spreading rate of rumors. On the other hand, in order 
to reduce the loss caused by emergencies, it is necessary to take timely and effective emergency strategies in dealing with rumor spreading. Such measures include holding a press conference and releasing relevant facts for emergencies, which can effectively prevent the spread of rumors.

Based on the study of the classic D-K model, this paper adds two factors, media coverage and emergency strategies by government, to the spread of rumors, analyzes the spread mechanism of the rumor under the influence of these two factors, and lays a theoretical foundation for further discussion of the control strategy of the rumors.

2.1. Model Hypothesis. (1) Based on the classical D-K model for the classification and propagation of the population, this paper further assumes that the ignorant propagates rumors with a certain probability when the ignorant meets the spreader, influenced by the media reports, and the propagation probability is gradually reduced, and finally a certain stability value is reached.

(2) When the rumor spreaders meet with the stifler, the spreader loses interest in the spreading; when the two spreaders meet, each of them loses the power to spread the rumor.

(3) Rumors spread in a certain range of communities, the total number of people is variable, and individuals entering the community do not know any information.

2.2. Formulation of the Rumor Model. According to the basic assumptions of the model, an open and mixed population in a certain area can be divided into 3 categories: the ignorant (nodes who have never heard rumors), the spreaders (nodes who know and spread rumors), and the stiflers (nodes who understand the truth of rumors and stop spreading rumors). Similar to the traditional models, at any time each and every node in propagation network is assumed to be in one of three possible categories. Let $I_{k}(t)\left(S_{k}(t), R_{k}(t)\right)$ denote the relative densities of ignorant (spreaders, stiflers) with degree $k$ at time $t$, respectively, with the normalization conditions $I_{k}(t)+S_{k}(t)+R_{k}(t)=1$. And in order to incorporate the dimensions of massive news coverage, we introduce the information variable $M(t)$ to describe the cumulative density of media coverage in other region at time $t$. This is because the contact between different types of individuals leads to the spread of rumors and then changes in the type of individuals, as shown in Figure 1.

Based on the idea of differential equation modeling, the $\mathrm{D}-\mathrm{K}$ model is further extended to establish a nonlinear dynamic model considering the impact of media coverage and emergency strategies by government. According to the above assumptions, one has the following system of $3 n+1$ ordinary differential equations (ODEs):

$$
\begin{aligned}
& \frac{d I_{k}(t)}{d t} \\
& =\Lambda \\
& \quad-\left(\beta_{1}-\beta_{2} \frac{k M(t)}{\alpha+k M(t)}\right) k I_{k}(t) \sum_{k^{\prime}} P\left(k^{\prime} \mid k\right) S_{k^{\prime}}(t)
\end{aligned}
$$

$$
-\eta I_{k}(t)
$$$$
\frac{d S_{k}(t)}{d t}
$$$$
=\left(\beta_{1}-\beta_{2} \frac{k M(t)}{\alpha+k M(t)}\right) k I_{k}(t) \sum_{k^{\prime}} P\left(k^{\prime} \mid k\right) S_{k^{\prime}}(t)
$$$$
-\eta S_{k}(t)-\delta S_{k}(t)
$$$$
-\varepsilon k S_{k}(t) \sum_{k^{\prime}} P\left(k^{\prime} \mid k\right)\left(S_{k^{\prime}}(t)+R_{k^{\prime}}(t)\right)
$$

$$
\begin{aligned}
& \frac{d R_{k}(t)}{d t} \\
& =\varepsilon k S_{k}(t) \sum_{k^{\prime}} P\left(k^{\prime} \mid k\right)\left(S_{k^{\prime}}(t)+R_{k^{\prime}}(t)\right)+\delta S_{k}(t) \\
& \quad-\eta R_{k}(t) \\
& \frac{d M(t)}{d t}=m_{0}+\omega \sum_{k^{\prime}} P(k) S_{k^{\prime}}(t)-\lambda M(t)
\end{aligned}
$$

where

$\Lambda$ : immigration rate, the individual enters a particular community at a constant rate;

$\alpha$ : the half-saturation constant, which reflects the impact of media coverage during rumor spreading;

$\eta$ : removal rate, each type of individual moves out of the group for some reason;

$\beta_{1}$ : the probability that the ignorant accepts and spreads rumors when the ignorant connects with the spreaders. $\beta_{1} I S$ indicates that some of the ignorants choose to trust the spreaders' information and enter the group;

$\beta_{2}$ : the maximum impact of media on the probability of transmission when ignorants are exposed to spreaders. $\beta_{2} k M /(\alpha+k M)$ expresses that the ignorant is in contact with the spreaders; the probability of the media's interference with the spread of rumor, with the increase of the number of spreaders, the intensities of the media, and the gradual increase in the media's interference in believing and disseminating untrue information to the individual, eventually tends to a maximum $\beta_{2}$, where $\alpha$ represents saturation coefficient and measures the degree of media coverage affecting rumor spreading. Considering that media coverage can slow down the spread and diffusion of rumor, it cannot completely prevent the spreading, so we assume that $\beta_{1} \geq \beta_{2}$;

$\varepsilon$ : stifling rate, the probability that a spreader transfers into a stifler when the spreader contacts with a spreader or a stifler; 


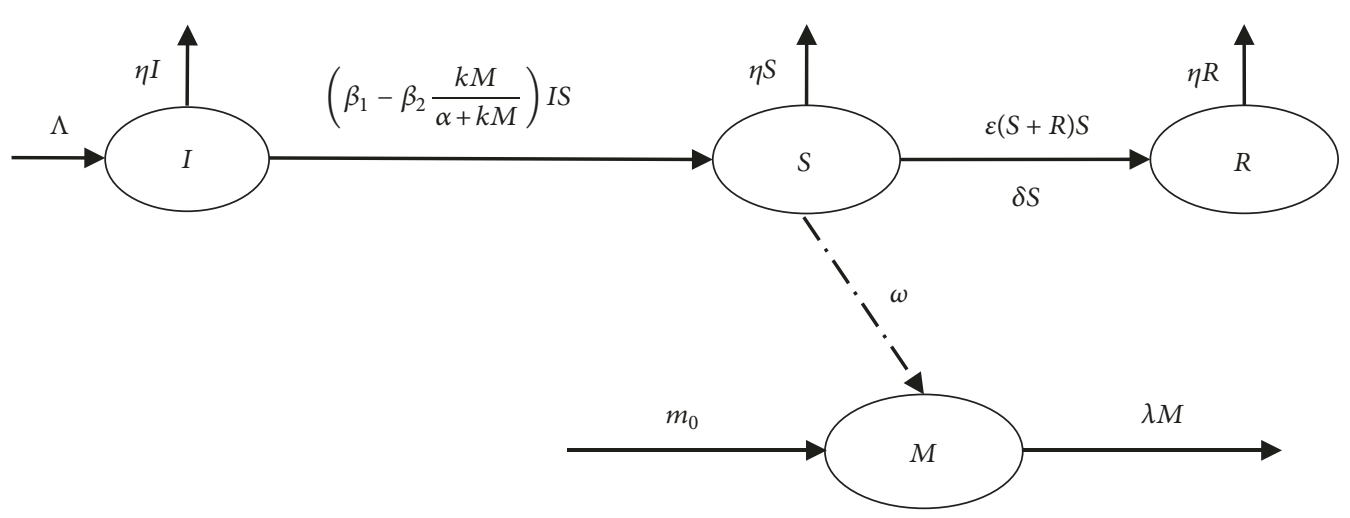

FIGURE 1: Schematic diagram of rumor spreading under the influence of media coverage and emergency strategies.

$\delta$ : emergency efficiency, by means of isolation and other compulsory administrative measures, the government loses the spreading ability of rumor spreaders;

$\omega$ : rate of media coverage being implemented, the influence is bidirectional. While the media is affecting rumor spreading, the development of rumors in turn affects the frequency of media coverage. And the parameter $\omega$ is a proportional constant with which media coverage is being implemented based on the current rumor spread;

$\lambda$ : depletion rate of media coverage due to ineffective implementation;

$m_{0}$ : density level of media coverage in this region based on the spread of rumor in another region. Even if there is no rumor case in the area, we must give warnings and nip the impossible situation in the bud.

According to the previous studies, $P\left(k^{\prime} \mid k\right)=$ $k^{\prime} P\left(k^{\prime}\right) /\langle k\rangle$ indicates the degree correlations so that a node of degree $k$ is connected to a node of degree $k^{\prime}$, and $\langle k\rangle=$ $\sum_{k} k P(k)$ is the average number of contacts each node can make. The media coverage function $k M(t) /(\alpha+k M(t))$ is similar to the Michaelis -Menten function $P_{1}(M)=C M(t) /(1+$ $D M(t)),(C, D \geq 0)$ (see $[34,35]$ and the references cited therein), which is a linear function of current prevalence of rumor and measures the impact of media coverage on a node in group $k$. It is related to the number of nodes he/she contacts and current density level of media coverage.

2.3. Model Analysis. This paper analyzes the mathematical model based on the stability related theory of differential equations, and due to the fact that the model depicts the dynamic propagation process of rumor in the crowd, the parameters involved are assumed to be nonnegative.

For the sake of simplicity, we set $\Theta(t)=\sum_{k^{\prime}} P\left(k^{\prime} \mid\right.$ $k) S_{k^{\prime}}(t)=(1 /\langle k\rangle) \sum_{k^{\prime}} k^{\prime} P\left(k^{\prime}\right) S_{k^{\prime}}(t)$ representing the probability that a randomly selected neighbor of a given node is a spreader while $\Phi(t)=\sum_{k^{\prime}} P\left(k^{\prime} \mid k\right)\left(R_{k^{\prime}}(t)\right)=$ $(1 /\langle k\rangle) \sum_{k^{\prime}} k^{\prime} P\left(k^{\prime}\right) R_{k^{\prime}}(t)$ denoting the probability that a randomly selected neighbor of a given node is a stifler. Then, system (1) can be rewritten as

$$
\begin{aligned}
& \frac{d S_{k}(t)}{d t} \\
& \quad=\left(\beta_{1}-\beta_{2} \frac{k M(t)}{\alpha+k M(t)}\right) k\left(1-S_{k}(t)-R_{k}(t)\right) \Theta(t) \\
& \quad-\eta S_{k}(t)-\delta S_{k}(t)-\varepsilon k S_{k}(t)(\Theta(t)+\Phi(t)) \\
& \frac{d R_{k}(t)}{d t}=\varepsilon k S_{k}(t)(\Theta(t)+\Phi(t))+\delta S_{k}(t)-\eta R_{k}(t) \\
& \frac{d M(t)}{d t}=m_{0}+\omega \sum_{k^{\prime}} P(k) S_{k^{\prime}}(t)-\lambda M(t)
\end{aligned}
$$

With no loss of generality, we assume that $\beta_{1}=\beta_{2}=\beta$, then the system (2) will be transformed into the following system (3).

$$
\begin{gathered}
\frac{\alpha \beta}{\alpha+k M(t)} k\left(1-S_{k}(t)-R_{k}(t)\right) \Theta(t)-\eta S_{k}(t) \\
-\delta S_{k}(t)-\varepsilon k S_{k}(t)(\Theta(t)+\Phi(t))=0 \\
\varepsilon k S_{k}(t)(\Theta(t)+\Phi(t))+\delta S_{k}(t)-\eta R_{k}(t)=0 \\
m_{0}+\omega \sum_{k^{\prime}} P(k) S_{k^{\prime}}(t)-\lambda M(t)=0
\end{gathered}
$$

Obviously, system (3) has a unique rumor-free equilibrium $E^{0}=\left(0,0, \ldots, 0, m_{0} / \lambda\right)_{1 \times(2 n+1)}$.

Furthermore, the existence of rumor spreading equilibrium point $E^{*}$ will be discussed. From system (3), a simple calculation shows that

$$
\begin{aligned}
& E^{*}=\left(S_{1}^{*}(t), S_{2}^{*}(t), \ldots, S_{n}^{*}(t), R_{1}^{*}(t), R_{2}^{*}(t), \ldots, R_{n}^{*}(t),\right. \\
& \left.\frac{\left(m_{0}+\omega \sum_{k} S_{k}^{*}(t) P(k)\right)}{\lambda}\right)_{1 \times(2 n+1)}
\end{aligned}
$$




$$
\begin{aligned}
& S_{k}^{*}(t)=\frac{\alpha \beta \eta k \Theta}{\mathscr{H}} \\
& R_{k}^{*}(t)=\alpha \beta \eta k \Theta \cdot \frac{(\delta+k \varepsilon(\Theta+\Phi))}{\mathscr{H}} \\
& M^{*}=\frac{\left(m_{0}+\omega \sum_{k} S_{k}^{*}(t) P(k)\right)}{\lambda}
\end{aligned}
$$

where $\mathscr{H}=\left(\alpha \eta+k \eta M^{*}+\alpha \beta k \Theta\right) \cdot(\eta+\delta+k \varepsilon(\Theta+\Phi))$.

Following the recipe of van den Driessche and Watmough [34], System (4) can be written as $d x / d t=\mathscr{F}(x)-v(x)$ and $x=\left(S_{k}, R_{k}\right)^{T}$, where $x_{1}, x_{2}, \ldots, x_{n}$ represent $S_{1}, S_{2}, \ldots, S_{n}$, and $x_{n+1}, x_{n+2}, \ldots, x_{2 n}$ denote $R_{1}, R_{2}, \ldots, R_{n}$, respectively. Applying the next generation matrix method in [34],

where the rate of appearance of new spreaders is

$$
\mathscr{F}(x)=\left(\begin{array}{c}
\frac{\alpha \beta k}{\alpha+k M(t)}\left(1-S_{k}(t)-R_{k}(t)\right) \Theta(t) \\
0
\end{array}\right)_{2 n \times 1}
$$

and the transfer rate of individuals out of compartments is

$$
\begin{gathered}
\mathscr{V}(x)=\left(\varepsilon k S_{k}(t)(\Theta(t)+\Phi(t))+\delta S_{k}(t)+\eta S_{k}(t)\right. \\
\left.-\varepsilon k S_{k}(t)(\Theta(t)+\Phi(t))-\delta S_{k}(t)+\eta R_{k}(t)\right)_{2 n \times 1}
\end{gathered}
$$

the Jacobian matrices of $\mathscr{F}(x)$ and $v(x)$ at the rumor-free equilibrium $E^{0}=\left(0,0, \ldots, 0, m_{0} / \lambda\right)_{1 \times(2 n+1)}$ are as follows:

$$
\begin{aligned}
& F=D \mathscr{F}\left(E^{0}\right)=\left(\begin{array}{cc}
F_{11} & 0 \\
0 & 0
\end{array}\right)_{2 n \times 2 n} \\
& V=D \mathscr{V}\left(E^{0}\right)=\left(\begin{array}{cc}
\delta+\eta & 0 \\
-\delta & \eta
\end{array}\right) E_{2 n \times 2 n}
\end{aligned}
$$

where

$$
\begin{aligned}
& F_{11}=\frac{\alpha \beta}{\langle k\rangle}\left(\begin{array}{cccc}
\frac{1}{\alpha+1 M(t)} 1 P(1) & \frac{1}{\alpha+1 M(t)} 2 P(2) & \cdots & \frac{1}{\alpha+1 M(t)} n P(n) \\
\frac{2}{\alpha+2 M(t)} 1 P(1) & \frac{2}{\alpha+2 M(t)} 2 P(2) & \cdots & \frac{2}{\alpha+2 M(t)} n P(n) \\
\vdots & \vdots & \ddots & \vdots \\
\frac{n}{\alpha+n M(t)} 1 P(1) & \frac{n}{\alpha+n M(t)} 2 P(2) & \cdots & \frac{n}{\alpha+n M(t)} n P(n)
\end{array}\right) \\
& V^{-1}=\left(\begin{array}{cc}
\frac{1}{\delta+\eta} & 0 \\
\frac{\delta}{\delta+\eta} & \frac{1}{\eta}
\end{array}\right) \cdot E_{2 n \times 2 n}
\end{aligned}
$$

According to the concept of next generation matrix and reproduction number given in [34], the reproduction number of system (2) equals $\mathfrak{R}_{0}=\rho\left(F V^{-1}\right)$, where $\rho\left(F V^{-1}\right)$ denotes the spectral radius of the matrix $F V^{-1}$. Then,

$$
\mathfrak{R}_{0}=\rho\left(F V^{-1}\right)=\frac{\alpha \beta \lambda}{(\eta+\delta)\langle k\rangle} \sum_{k=1}^{n} \frac{k^{2} P(k)}{\alpha \lambda+k m_{0}}
$$

The basic reproductive number $\mathfrak{R}_{0}$ describes the average number of new spreaders at the beginning of the spreading process. And according to [34], if $\mathfrak{R}_{0}<1$, then there is no more rumor to spread in the networks; while if $\mathfrak{R}_{0}>1$, then the rumor will persist in the networks.

\section{Optimal Control Strategies of the Model}

Based on the analysis of the previous section, we find that it is not enough for rumor management to discuss the dynamic process of rumor, and the optimal control techniques are of great use in optimal strategies to control various kinds of rumors. Based on the optimal control theory and numerical simulation method, we further expand the system (1) to explore the impact of the two factors such as media coverage and emergency strategies on the final spread of rumor.

In real life, when rumors break out, some control measures will be taken by officials including precontrol measures (such as popularization of science education, Microblog hot tweens, press conference, and other media coverage measures) and postcontrol measures (by means of isolating and compulsive punishments of spreaders to transform them into stiflers).

On one hand, when rumors prevail, the media should increase the broadcast to suppress them; on the other hand, when the rumor is controlled, the amount of media coverage should be reduced accordingly. So, we assume media coverage is a dynamically adjusted process with the density of the spreaders in the current environment (the range of rumor spreading). Meanwhile, when there are mass spreaders in the environment, a series of emergency strategies by government must be taken to reduce the harm of rumors, such as forced isolation, enhanced punishment, and cut off sources of rumors spreading. But taking into account the control costs 
and other factors, the emergency strategies by government can be slackened a little when the rumor situation slows down. From this perspective, emergency measures are also a dynamic adjustment process.

In the actual model, we select the Lebesgue squareintegrable control functions $u_{m}(t), u_{\delta}(t)$ to represent the control variables of media coverage and emergency strategies by government, respectively, and the system (1) can be rewritten as a new control system:

$$
\begin{aligned}
& \frac{d I_{k}(t)}{d t}=\Lambda-\left(\beta_{1}-\beta_{2} \frac{k M(t)}{\alpha\left(1-u_{m}(t)\right)+k M(t)}\right) \\
& \cdot k I_{k}(t) \sum_{k^{\prime}} P\left(k^{\prime} \mid k\right) S_{k^{\prime}}(t)-\eta I_{k}(t) \\
& \frac{d S_{k}(t)}{d t}=\left(\beta_{1}-\beta_{2} \frac{k M(t)}{\alpha\left(1-u_{m}(t)\right)+k M(t)}\right) k I_{k}(t) \\
& \cdot \sum_{k^{\prime}} P\left(k^{\prime} \mid k\right) S_{k^{\prime}}(t)-\eta S_{k}(t)-\delta\left(1-u_{\delta}(t)\right) S_{k}(t) \\
& -\varepsilon k S_{k}(t) \sum_{k^{\prime}} P\left(k^{\prime} \mid k\right)\left(S_{k^{\prime}}(t)+R_{k^{\prime}}(t)\right) \\
& \frac{d R_{k}(t)}{d t}=\varepsilon k S_{k}(t) \sum_{k^{\prime}} P\left(k^{\prime} \mid k\right)\left(S_{k^{\prime}}(t)+R_{k^{\prime}}(t)\right) \\
& +\delta\left(1-u_{\delta}(t)\right) S_{k}(t)-\eta R_{k}(t)
\end{aligned}
$$

$$
\begin{array}{r}
\frac{d M(t)}{d t}=m_{0}+\omega \sum_{k^{\prime}} P(k) S_{k^{\prime}}(t)-\lambda M(t) \\
\quad k=1,2, \ldots, n
\end{array}
$$

with initial conditions

$$
\begin{aligned}
& I_{k}(0)=I_{k 0} \geq 0, \\
& S_{k}(0)=S_{k 0} \geq 0, \\
& R_{k}(0)=R_{k 0} \geq 0, \\
& \left(I_{1}(0), \ldots, I_{n}(0), S_{1}(0), \ldots, S_{n}(0), R_{1}(0), \ldots, R_{n}(0)\right)^{T} \in \widetilde{\Omega}
\end{aligned}
$$

where $\widetilde{\Omega}=\left\{\left(I_{1}, \ldots, I_{n}, S_{1}, \ldots, S_{n}, R_{1}, \ldots, R_{n}\right)^{T} \in \mathbb{R}_{+}^{3 n} \mid I_{k}+\right.$ $\left.S_{k}+R_{k}=1, k=1,2, \ldots, n\right\}$.

The objective of optimal control is to minimize the negative effects of rumor dissemination, in the meantime, and maximize the positive social utility. This paper selects the following aspects to consider the issue of social utility of rumor spreading:

(1) Maximize the number of ignorants and stiflers.

(2) Try to minimize the number of rumor spreaders.

(3) Minimize the controlling costs of variable control in the process.

Now, we choose an objective function to be

$$
\max J\left(u_{m}, u_{\delta}\right)=\int_{0}^{t_{f}} \sum_{k}\left[A_{k}\left(I_{k}(t)+R_{k}(t)\right)-B_{k} S_{k}(t)-C_{k}\left(u_{k}^{2}(t)+u_{k}^{2}(t)\right)\right] d t
$$

where $\left[0, t_{f}\right]$ denotes the time period during a control strategy that is imposed on system (13). The parameters $A_{k}, B_{k}, C_{k}$ are the weight coefficients of the nonspreading group, spreading group and process control and can be regarded as the balance coefficients between different variables in time $t_{f}$, and all social utility is converted into a function with the goal of cost. $u_{m}(t)=u_{m}^{\max }, u_{\delta}(t)=u_{\delta}^{\max }$ represent the intensity of media coverage and emergency strategies by government, respectively; it has the greatest impact on the system. $C_{k} u_{m}^{2}, C_{k} u_{\delta}^{2}$ represent the cost of media coverage and emergency strategies by government, respectively. Since then, we can take the Lagrangian as

$$
\begin{aligned}
& L\left(I_{k}(t), S_{k}(t), R_{k}(t), u_{m}, u_{\delta}\right) \\
& \quad=A_{k}\left(I_{k}(t)+R_{k}(t)\right)-B_{k} S_{k}(t)-C_{k}\left(u_{m}^{2}+u_{\delta}^{2}\right)
\end{aligned}
$$

The optimal control variable $u_{m}(t), u_{\delta}(t)$ is determined by the following equation:

$$
J\left(u_{m}^{*}, u_{\delta}^{*}\right)=\max \left\{J\left(u_{m}, u_{\delta}\right) \mid u_{m}, u_{\delta} \in U\right\}
$$

where $U=\left\{\left(u_{m}, u_{\delta}\right) \mid\right.$ measurable : $0 \leq u_{m} \leq$ $\left.1,0 \leq u_{\delta} \leq 1, t \in\left[0, t_{f}\right], k=1,2, \ldots, n\right\}$ is the control set, $u_{m}(t), u_{\delta}(t)$ are the measurable variables, and $\left[0, t_{f}\right]$ expresses the time period during which a control strategy is imposed on system (13), which indicates an admissible control.

According to the conclusion of the existence of the optimal control solution by Fleming and Rishel [35], Theorem 1 demonstrates the existence of the optimal control solution in detail.

Theorem 1. Under the limit of (17), system (13) has an optimal control $u_{m}^{*}, u_{\delta}^{*}$, that is, $\max \left\{J\left(u_{m}, u_{\delta}\right) \mid u_{m}, u_{\delta} \in U\right\}=$ $J\left(u_{m}^{*}, u_{\delta}^{*}\right)$.

Proof. In order to prove the conclusion of Theorem 1, according to the theory of Fleming and Rishel [35] (Theorem 4.1, PP 68-69), the following conditions must be satisfied:

(1) The control set and corresponding state variable are not empty.

(2) The control set is a closed set of convexity by definition.

(3) The upper bound of the solution of system (13) is a linear function. Each right-hand side of the state system is continuous and is bounded by a sum of the bounded 
control and the state. Furthermore, it can be written as a linear function of the control variate $u_{m}(t), u_{\delta}(t)$ with coefficients depending on time and state.

(4) The integral of the system (13) is concave function in the control set, $c_{2}-c_{1}\left(\left|u_{m}\right|^{k}+\left|u_{\delta}\right|^{k}\right)$ is the upper bound of the integral function, and $c_{2}>0, c_{1}>0, k>1$.

According to Theorem 9.2.1 of Lukes [36], the points on the boundary of system (13) are within the control set. It is known from definition that control set $U$ is a closed set of convexity. According to Theorem 1, the solution of the system (13) has the supremum and satisfies condition (3); the item $A_{k}\left(I_{k}(t)+R_{k}(t)\right)-B_{k} S_{k}(t)-C_{k}\left(u_{m}^{2}+u_{\delta}^{2}\right)$ in the objective function is a concave function on the control set $U$, and there are some constants $c_{2}>0, c_{1}>0, k>1$ making

$$
\begin{aligned}
& A_{k}\left(I_{k}(t)+R_{k}(t)\right)-B_{k} S_{k}(t)-C_{k}\left(u_{m}^{2}+u_{\delta}^{2}\right) \\
& \quad \leq c_{2}-c_{1}\left(\left|u_{m}\right|^{k}+\left|u_{\delta}\right|^{k}\right)
\end{aligned}
$$

Then system (13) satisfies all four conditions and has optimal control. That is, there exist $u_{m}^{*}, u_{\delta}^{*}$ making $\max \left\{J\left(u_{m}, u_{\delta}\right) \mid u_{m}, u_{\delta} \in U\right\}=J\left(u_{m}^{*}, u_{\delta}^{*}\right)$ set up. This completes the proof.

Theorem 1 proves the existence of optimal control under the condition of the restrictive system (13). We discuss the necessary conditions for optimal control by means of the Pontryagin Maximum Principle [37].

Pontryagin Maximum Principle requires the use of auxiliary functions to explore the optimal value problem. The differential equation system (13) will be used as the constraint condition of the objective function. After discussing the existence of the optimal control, we use Pontryagin Maximum Principle to solve the auxiliary variables of the differential equation, the corresponding boundedness condition, and the properties of the control variables $u_{m}(t), u_{\delta}(t)$.

Based on the above analysis, the Hamiltonian for the control problem is constructed as

$$
\begin{aligned}
H & \left(I_{k}(t), S_{k}(t), R_{k}(t), u_{m}(t), u_{\delta}(t), \lambda_{1 k}(t), \lambda_{2 k}(t), \lambda_{3 k}(t), \lambda_{4 k}(t)\right) \\
& =L\left(I_{k}(t), S_{k}(t), R_{k}(t), u_{m}(t), u_{\delta}(t)\right) \\
& +\sum_{k=1}^{n}\left[\lambda_{1 k}(t) \frac{I_{k}(t)}{d t}+\lambda_{2 k}(t) \frac{S_{k}(t)}{d t}+\lambda_{3 k}(t) \frac{R_{k}(t)}{d t}+\lambda_{4 k}(t) \frac{M(t)}{d t}\right] \\
& =L\left(I_{k}(t), S_{k}(t), R_{k}(t), M(t), u_{m}(t), u_{\delta}(t)\right) \\
& +\sum_{k=1}^{n}\left(\lambda_{1 k}(t)\left(\Lambda-\left(\beta_{1}-\beta_{2} \frac{k M\left(1-u_{m}(t)\right)+k M}{\alpha}\right) k I_{k}(t) \sum_{k^{\prime}} P\left(k^{\prime} \mid k\right) S_{k^{\prime}}(t)-\eta I_{k}(t)\right)\right. \\
& +\lambda_{2 k}(t)\left(\left(\beta_{1}-\beta_{2} \frac{k M\left(1-u_{m}(t)\right)+k M}{\alpha}\right) k I_{k}(t) \sum_{k^{\prime}} P\left(k^{\prime} \mid k\right) S_{k^{\prime}}(t)\right. \\
& +\eta S_{k}(t)-\delta\left(1-u_{\delta}(t)\right) S_{k}(t) \\
& \left.+\lambda_{4 k}(t)\left(m_{0}+\omega \sum_{k^{\prime}} P(k) S_{k^{\prime}}(t)-\lambda M(t)\right)\right) \\
& \left.+\varepsilon k S_{k}(t) \sum_{k^{\prime}} P\left(k^{\prime} \mid k\right)\left(S_{k^{\prime}}(t)+R_{k^{\prime}}(t)\right)\right) \\
& +\lambda_{3 k}(t)\left(\varepsilon k S_{k}(t) \sum_{k^{\prime}} P\left(k^{\prime} \mid k\right)\left(S_{k^{\prime}}(t)+R_{k^{\prime}}(t)\right)\right.
\end{aligned}
$$

where $\lambda_{1 k}(t), \lambda_{2 k}(t), \lambda_{3 k}(t), \lambda_{4 k}(t)$ are the adjoint functions to be determined suitable, to simplify, with $\lambda_{1 k}, \lambda_{2 k}, \lambda_{3 k}, \lambda_{4 k}$, respectively.
Next, we will derive the necessary conditions for the optimal control strategy by means of the Pontryagin Maximum Principle [37]. 
Theorem 2. Let $I_{k}^{*}(t), S_{k}^{*}(t), R_{k}^{*}(t), M^{*}(t)$ be optimal state solutions associated with the optimal control variable $u_{m}^{*}(t), u_{\delta}^{*}(t)$ for the controlled system (13) with the corresponding initial conditions, where $k=1,2, \ldots, n$. Then, there exist adjoint functions $\lambda_{1 k}(t), \lambda_{2 k}(t), \lambda_{3 k}(t)$, and $\lambda_{4 k}(t)$, which satisfy

$$
\begin{aligned}
& \frac{d \lambda_{1 k}(t)}{d t}=-A_{k}+\lambda_{1 k}(t)\left(\frac{\alpha \beta k \Theta}{\alpha\left(1-u_{m}(t)\right)+k M}-\eta\right) \\
& -\lambda_{2 k}(t) \frac{\alpha \beta k \Theta}{\alpha\left(1-u_{m}(t)\right)+k M} \\
& \frac{d \lambda_{2 k}(t)}{d t}=B_{k}+\lambda_{1 k}(t) \frac{\alpha \beta k^{2} P(k) I_{k}(t)}{\left(\alpha\left(1-u_{m}(t)\right)+k M\right)\langle k\rangle} \\
& -\lambda_{2 k}(t)\left(\frac{\alpha \beta k^{2} P(k) I_{k}(t)}{\left(\alpha\left(1-u_{m}(t)\right)+k M\right)\langle k\rangle}\right. \\
& -\varepsilon k(\Theta(t)+\Phi(t))-\varepsilon k S_{k}(t) \frac{k P(k)}{\langle k\rangle}-\eta \\
& \left.-\delta\left(1-u_{\delta}(t)\right)\right)-\lambda_{3 k}(t)(\varepsilon k(\Theta(t)+\Phi(t)) \\
& \left.+\varepsilon k S_{k}(t) \frac{k P(k)}{\langle k\rangle}+\delta\left(1-u_{\delta}(t)\right)\right) \\
& \frac{d \lambda_{3 k}(t)}{d t}=-A_{k}+\lambda_{2 k}(t) \varepsilon k S_{k}(t) \frac{k P(k)}{\langle k\rangle}-\lambda_{3 k}(t) \\
& \cdot\left(\varepsilon k S_{k}(t) \frac{k P(k)}{\langle k\rangle}+\eta\right) \\
& \frac{d \lambda_{4 k}(t)}{d t}=\lambda_{1 k}(t) \frac{\alpha \beta k^{2} I_{k}(t) \Theta}{\left(\alpha\left(1-u_{m}(t)\right)+k M\right)^{2}}-\lambda_{4 k}(t) \\
& \cdot \lambda
\end{aligned}
$$

with transversality conditions,

$$
\begin{aligned}
\lambda_{1 k}\left(t_{f}\right)=\lambda_{2 k}\left(t_{f}\right)=\lambda_{3 k}\left(t_{f}\right)=\lambda_{4 k}\left(t_{f}\right) & =0, \\
k & =1,2, \ldots, n
\end{aligned}
$$

Furthermore, the optimal control is given as follows:

$$
\begin{aligned}
& u_{m}^{*}(t) \\
& =\min \left\{\max \left\{0, \frac{\alpha \beta\left(\lambda_{1 k}-\lambda_{2 k}\right)}{2 C_{k}\left(\left(1-u_{m}\right) \alpha+k M\right)^{2}}\right\}, u_{m}^{\max }\right\}
\end{aligned}
$$

$$
u_{\delta}^{*}(t)=\min \left\{\max \left\{0, \frac{\delta S_{k}(t)\left(\lambda_{2 k}-\lambda_{3 k}\right)}{2 C_{k}}\right\}, u_{\delta}^{\max }\right\}
$$

and $k=1,2, \ldots, n$.

Proof. To determine the adjoint equations and the transversality conditions, we use the Hamiltonian (5.6). By using the necessary condition for optimal control problems and differentiating the Hamiltonian (5.6) with $I_{k}(t), S_{k}(t)$, and $R_{k}(t)$ at

$$
\begin{aligned}
\Delta & =\left\{I_{k}(t), S_{k}(t), R_{k}(t), M(t), u_{m}(t), u_{\delta}(t) \mid I_{k}(t)\right. \\
& =I_{k}^{*}(t), S_{k}(t)=S_{k}^{*}(t), R_{k}(t)=R_{k}^{*}(t), M(t) \\
& \left.=M^{*}(t), u_{m}(t)=u_{m}^{*}, u_{\delta}(t)=u_{\delta}^{*}\right\}
\end{aligned}
$$

we obtain

$$
\begin{gathered}
\frac{d \lambda_{1 k}(t)}{d t}=-\frac{\partial}{\partial I_{k}(t)} H\left(I_{k}(t), S_{k}(t), R_{k}(t), M(t),\right. \\
\left.u_{m}(t), u_{\delta}(t), \lambda_{1 k}(t), \lambda_{2 k}(t), \lambda_{3 k}(t), \lambda_{4 k}(t)\right)\left.\right|_{\Delta} \\
\frac{d \lambda_{2 k}(t)}{d t}=-\frac{\partial}{\partial S_{k}(t)} H\left(I_{k}(t), S_{k}(t), R_{k}(t), M(t),\right. \\
\left.u_{m}(t), u_{\delta}(t), \lambda_{1 k}(t), \lambda_{2 k}(t), \lambda_{3 k}(t), \lambda_{4 k}(t)\right)\left.\right|_{\Delta} \\
\frac{d \lambda_{3 k}(t)}{d t}=-\frac{\partial}{\partial R_{k}(t)} H\left(I_{k}(t), S_{k}(t), R_{k}(t), M(t),\right. \\
\left.u_{m}(t), u_{\delta}(t), \lambda_{1 k}(t), \lambda_{2 k}(t), \lambda_{3 k}(t), \lambda_{4 k}(t)\right)\left.\right|_{\Delta} \\
\frac{d \lambda_{4 k}(t)}{d t}=-\frac{\partial}{M(t)} H\left(I_{k}(t), S_{k}(t), R_{k}(t), M(t),\right. \\
\left.u_{m}(t), u_{\delta}(t), \lambda_{1 k}(t), \lambda_{2 k}(t), \lambda_{3 k}(t), \lambda_{4 k}(t)\right)\left.\right|_{\Delta}
\end{gathered}
$$

where $k=1,2, \ldots, n$.

Hamiltonian minimizing condition: at the interior points, we have

$$
\frac{\partial}{\partial u_{m}(t)} H\left(I_{k}(t), S_{k}(t), R_{k}(t), M(t), u_{m}(t), u_{\delta}(t),\right.
$$

$$
\begin{aligned}
& \left.\lambda_{1 k}(t), \lambda_{2 k}(t), \lambda_{3 k}(t), \lambda_{4 k}(t)\right)\left.\right|_{\Delta}=-2 C_{k} u_{m} \\
& +\frac{\alpha \beta\left(\lambda_{1 k}-\lambda_{2 k}\right)}{\left(\left(1-u_{m k}\right) \alpha+k M\right)^{2}}=0
\end{aligned}
$$

$$
\begin{aligned}
& \frac{\partial}{\partial u_{\delta}(t)} H\left(I_{k}(t), S_{k}(t), R_{k}(t), M(t), u_{m}(t), u_{\delta}(t),\right. \\
& \left.\lambda_{1 k}(t), \lambda_{2 k}(t), \lambda_{3 k}(t), \lambda_{4 k}(t)\right)\left.\right|_{\Delta}=-2 C_{k} u_{\delta}+\delta S_{k}(t) \\
& \cdot\left(\lambda_{2 k}-\lambda_{3 k}\right)=0
\end{aligned}
$$

which implies 


$$
\begin{aligned}
u_{m}^{*}(t) & =\left\{\begin{array}{lc}
0, & \frac{\alpha \beta\left(\lambda_{1 k}-\lambda_{2 k}\right)}{2 C_{k}\left(\left(1-u_{m}\right) \alpha+k M\right)^{2}}<0 \\
\frac{\alpha \beta\left(\lambda_{1 k}-\lambda_{2 k}\right)}{2 C_{k}\left(\left(1-u_{m}\right) \alpha+k M\right)^{2}}, & 0 \leq \frac{\alpha \beta\left(\lambda_{1 k}-\lambda_{2 k}\right)}{2 C_{k}\left(\left(1-u_{m}\right) \alpha+k M\right)^{2}} \leq 1 \\
u_{m}^{\max } & \frac{\alpha \beta\left(\lambda_{1 k}-\lambda_{2 k}\right)}{2 C_{k}\left(\left(1-u_{m}\right) \alpha+k M\right)^{2}}>1
\end{array}\right. \\
u_{\delta}^{*}(t) & = \begin{cases}0, & \frac{\delta S_{k}(t)\left(\lambda_{2 k}-\lambda_{3 k}\right)}{2 C_{k}}<0 \\
\frac{\delta S_{k}(t)\left(\lambda_{2 k}-\lambda_{3 k}\right)}{2 C_{k}}, & 0 \leq \frac{\delta S_{k}(t)\left(\lambda_{2 k}-\lambda_{3 k}\right)}{2 C_{k}} \leq 1 \\
u_{\delta}^{\max }, & \frac{\delta S_{k}(t)\left(\lambda_{2 k}-\lambda_{3 k}\right)}{2 C_{k}} \geq 1\end{cases}
\end{aligned}
$$

that is,

$$
\begin{aligned}
& u_{m}^{*}(t) \\
& =\min \left\{\max \left\{0, \frac{\alpha \beta\left(\lambda_{1 k}-\lambda_{2 k}\right)}{2 C_{k}\left(\left(1-u_{m}\right) \alpha+k M\right)^{2}}\right\}, u_{m}^{\max }\right\} \\
& u_{\delta}^{*}(t)=\min \left\{\max \left\{0, \frac{\delta S_{k}(t)\left(\lambda_{2 k}-\lambda_{3 k}\right)}{2 C_{k}}\right\}, u_{\delta}^{\max }\right\}
\end{aligned}
$$

$k=1,2, \ldots, n$

The transversality conditions are obvious, namely,

$$
\lambda_{1 k}\left(t_{f}\right)=\lambda_{2 k}\left(t_{f}\right)=\lambda_{3 k}\left(t_{f}\right)=0, \quad k=1,2, \ldots, n
$$

This completes the proof.

Therefore, according to the analysis above, we can easily obtain the following optimality system:

$$
\begin{aligned}
& \frac{d I_{k}(t)}{d t}=\Lambda-\frac{\alpha \beta k}{\alpha\left(1-u_{m}^{*}\right)+k M} k I_{k}(t) \sum_{k^{\prime}} P\left(k^{\prime} \mid k\right) \\
& \cdot S_{k^{\prime}}(t)-\eta I_{k}(t) \\
& \frac{d S_{k}(t)}{d t}=\frac{\alpha \beta k}{\alpha\left(1-u_{m}^{*}\right)+k M} k I_{k}(t) \sum_{k^{\prime}} P\left(k^{\prime} \mid k\right) S_{k^{\prime}}(t) \\
& -\eta S_{k}(t)-\delta\left(1-u_{\delta}^{*}\right) S_{k}(t)-\varepsilon k S_{k}(t) \sum_{k^{\prime}} P\left(k^{\prime} \mid k\right) \\
& \cdot\left(S_{k^{\prime}}(t)+R_{k^{\prime}}(t)\right) \\
& \frac{d R_{k}(t)}{d t}=\varepsilon k S_{k}(t) \sum_{k^{\prime}} P\left(k^{\prime} \mid k\right)\left(S_{k^{\prime}}(t)+R_{k^{\prime}}(t)\right) \\
& \quad+\delta\left(1-u_{\delta}^{*}\right) S_{k}(t)-\eta S_{k}(t) \\
& \frac{d M(t)}{d t}=m_{0}+\omega \sum_{k^{\prime}} P(k) S_{k^{\prime}}(t)-\lambda M(t)
\end{aligned}
$$

$$
\begin{aligned}
& \frac{d \lambda_{1 k}(t)}{d t}=A_{k}+\lambda_{1 k}(t)\left(\frac{\alpha \beta k \Theta}{\alpha\left(1-u_{m}\right)+k M}-\eta\right) \\
& -\lambda_{2 k}(t) \frac{\alpha \beta k \Theta}{\alpha\left(1-u_{m}\right)+k M} \\
& \frac{d \lambda_{2 k}(t)}{d t}=B_{k}+\lambda_{1 k}(t) \frac{\alpha \beta k^{2} P(k) I_{k}(t)}{\left(\alpha\left(1-u_{m}\right)+k M\right)\langle k\rangle} \\
& -\lambda_{2 k}(t)\left(\frac{\alpha \beta k^{2} P(k) I_{k}(t)}{\left(\alpha\left(1-u_{m}\right)+k M\right)\langle k\rangle}\right. \\
& -\varepsilon k(\Theta(t)+\Phi(t))-\varepsilon k S_{k}(t) \frac{k P(k)}{\langle k\rangle}-\eta \\
& \left.-\delta\left(1-u_{\delta}\right)\right)-\lambda_{3 k}(t)(\varepsilon k(\Theta(t)+\Phi(t)) \\
& \left.+\varepsilon k S_{k}(t) \frac{k P(k)}{\langle k\rangle}+\delta\left(1-u_{\delta}\right)\right) \\
& \frac{d \lambda_{3 k}(t)}{d t}=-A_{k}+\lambda_{2 k}(t) \varepsilon k S_{k}(t) \frac{k P(k)}{\langle k\rangle}-\lambda_{3 k}(t) \\
& \left(\varepsilon k S_{k}(t) \frac{k P(k)}{\langle k\rangle}+\eta\right)
\end{aligned}
$$

where $u_{m}(t)$ and $u_{\delta}(t)$ are given by (27)-(30), respectively. The initial conditions are $I_{k}(0)=I_{0}, S_{k}(0)=S_{0}, R_{k}(0)=R_{0}$, $\lambda_{i k}\left(t_{f}\right)=0, i=1,2,3 . k=1,2, \ldots, n$. Obviously, the differential equation system satisfies the Lipschitz continuity condition, and then we get the only optimal control variable $u_{m}^{*}(t)$ and $u_{\delta}^{*}(t)$.

\section{Numerical Simulations}

To complement the mathematical analysis carried out in the previous section, a series of numerical simulations were performed to support and extend our theoretical results on BA scale-free networks $\left(p(k)=2 m^{2} k^{-3}, m=3\right)$. In the first part, we analyze the sensitivity of the basic reproductive number $\mathfrak{R}_{0}$ in terms of the model parameters. During the 


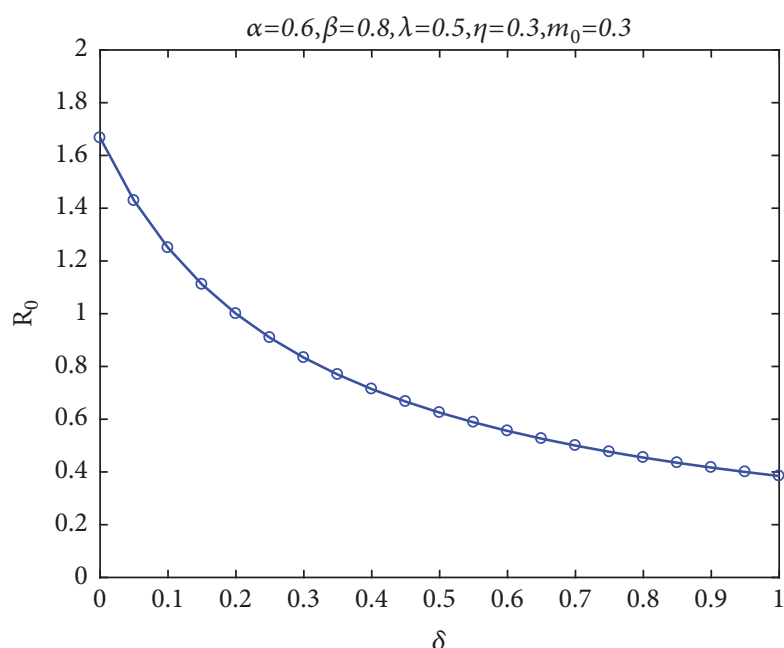

(a)

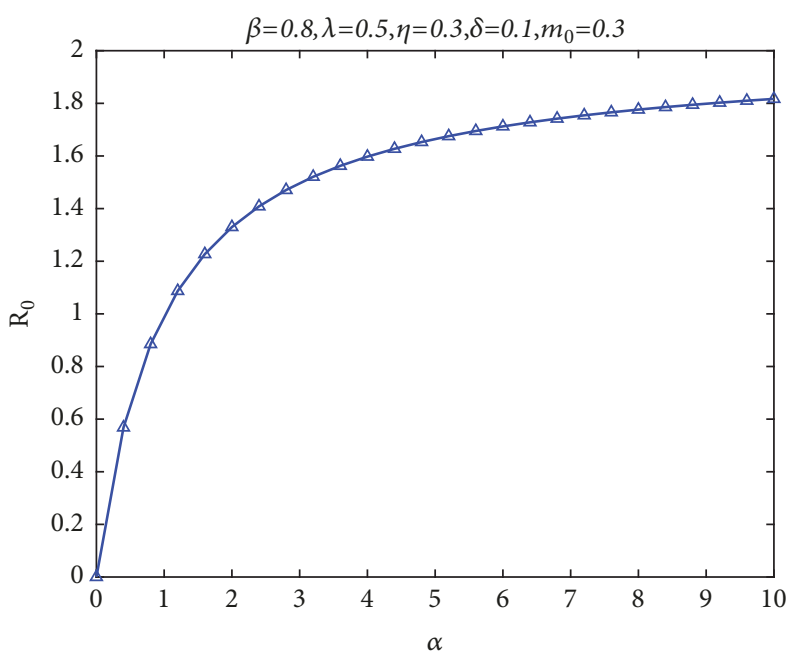

(c)

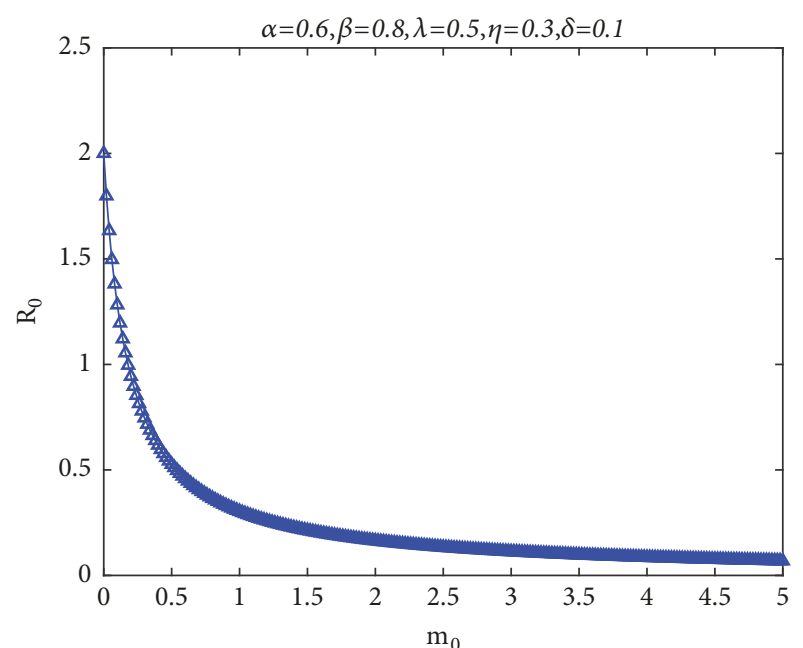

(b)

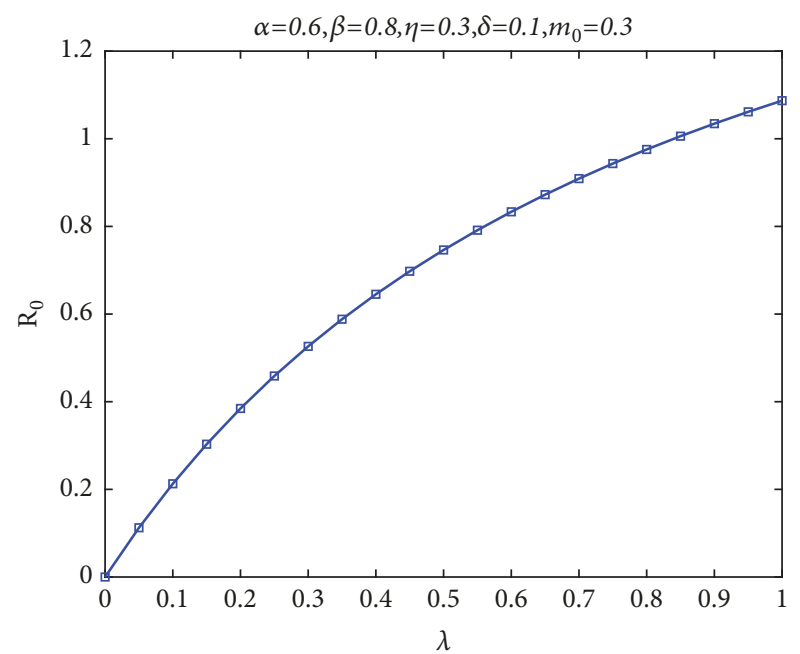

(d)

FIGURE 2: The relationship between the basic reproduction number $\mathfrak{R}_{0}$ and the model parameters $\delta, m_{0}, \alpha$, and $\lambda$ on BA scale-free network.

second part, we simulated and analyzed the model involving optimal control.

From Figure 2, it is easy to see that the basic reproduction number $\mathfrak{R}_{0}$ decreases with increasing the model parameters $\delta$ and $m_{0}$, expressed as inhibition of spreading, while it increases with the increase of the parameters $\alpha$ and $\lambda$, expressed as promotion of spreading. Comparing the first two pictures, we can see that the influence of $m_{0}$ on $\mathfrak{R}_{0}$ is greater than that of $\delta$, which shows that prior media coverage is more effective than the postcontrol from the government. From the last two pictures, it is known that the influence of $\alpha$ on $\mathfrak{R}_{0}$ is less than that of $\lambda$, which means that we should improve the effectiveness of campaigning (i.e., lower the depletion of media coverage $\lambda$ ) to reduce $\mathfrak{R}_{0}$.

First, the dynamic propagation process of the rumor without the optimal control is discussed. Figure 3 depicts the total densities of rumor spreaders changing over time with $\mathfrak{R}_{0}<1$, while Figure 4 with $\mathfrak{R}_{0}>1$. From these two figures, it can be found that when the corresponding parameter is adjusted to make $\mathfrak{R}_{0}>1$, the rumor propagator tends to a stable value. The rumor continues to spread and causes bad social influence. When the corresponding parameter is adjusted to make $\mathfrak{R}_{0}<1$, the rumor spreaders will be reduced to 0 , and the rumor will be well controlled.

The influence of different values $\delta$ on the total density of spreaders is shown in Figures 3 and 4 . We can see that the bigger the value of $\delta$ is (the more effective of the emergency strategies control means), the lower the total density of infected nodes is. The same operation is done for the parameters $m_{0}, \alpha$, and $\lambda$. From Figures 3(b) and 4(b), one obtains that the larger the value of $m_{0}$ is, the more easily the rumor could be governed, which means that the rumor control and government center had better timely report when the rumor emerges in another neighboring region owing to rapid spatial spread, although there is no spreader found in the present region. From Figures 3(c) and 4(c), we can obtain that the larger the value of $\alpha$ is, the more difficult the rumor could be governed. And Figures 3(d) and 4(d) illustrate how 


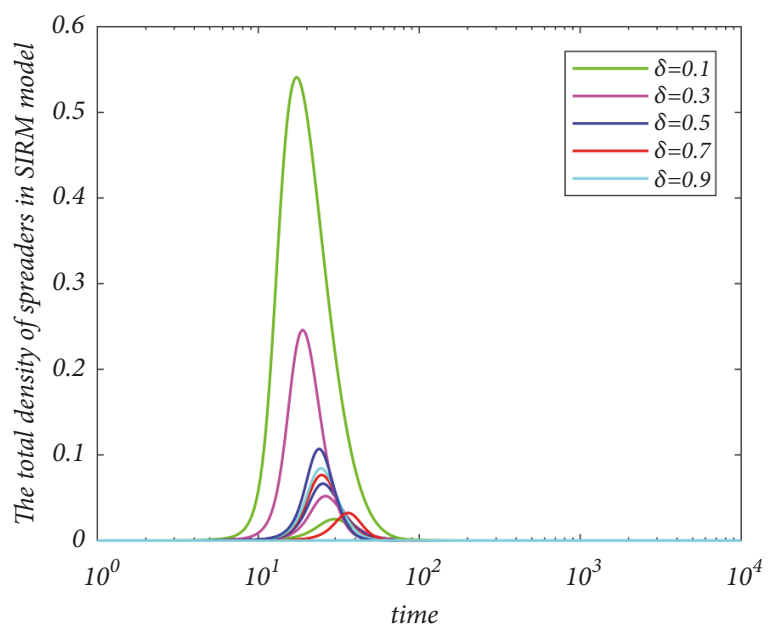

(a)

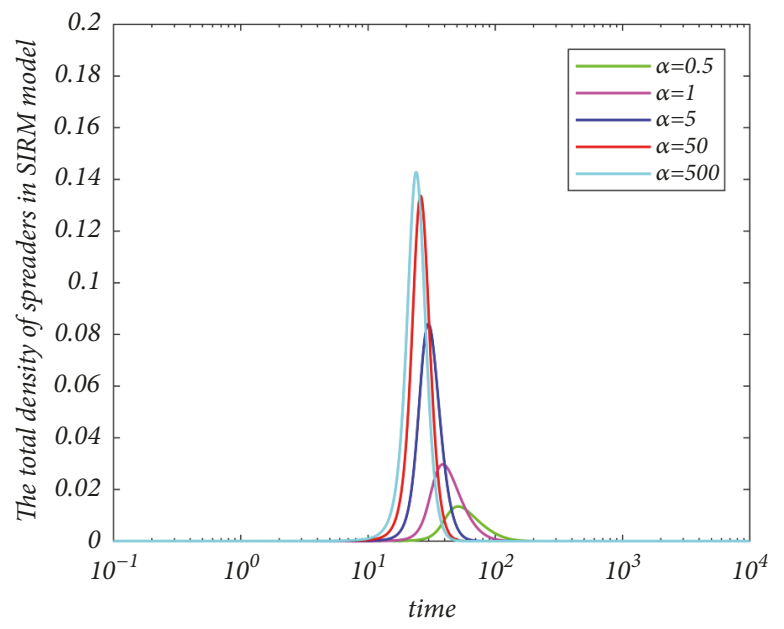

(c)

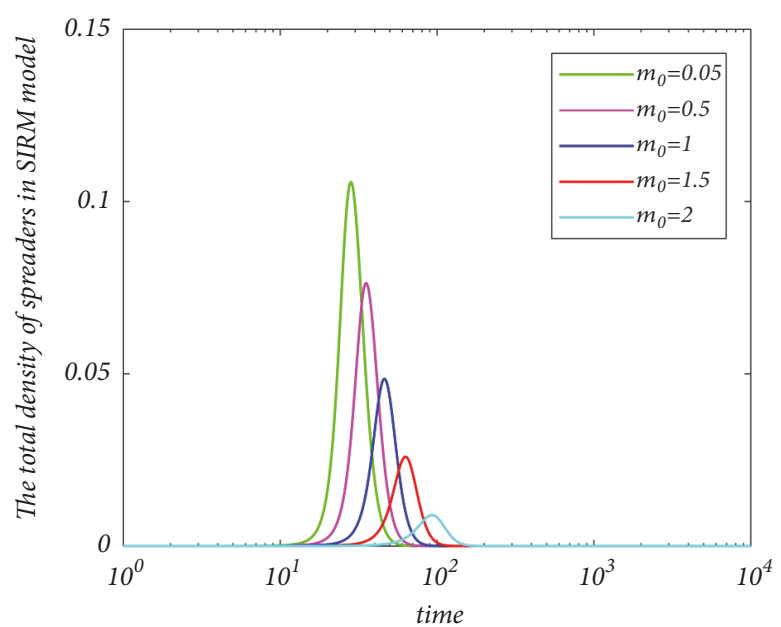

(b)

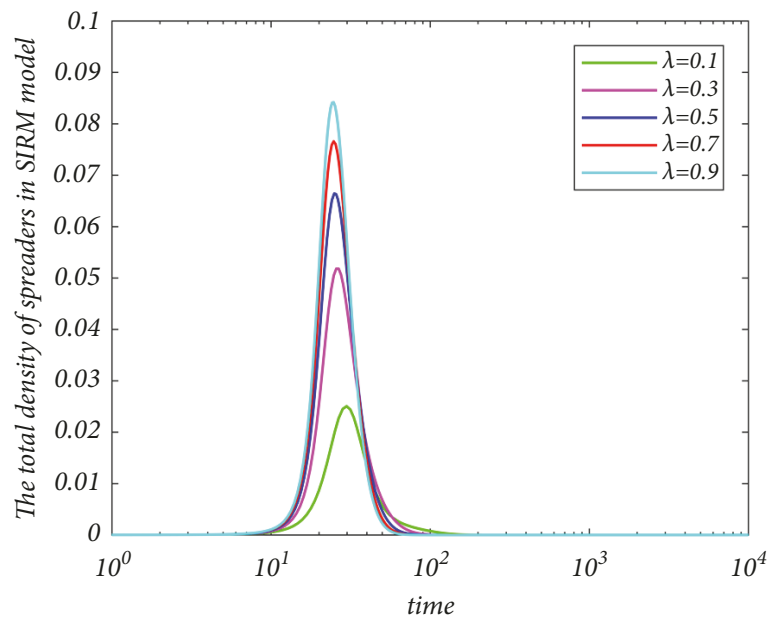

(d)

FIgURE 3: The impact of different values of $\delta, m_{0}, \alpha, \lambda$ on the total density of spreaders when $\Re_{0}<1$.

the densities of spreaders change over time with different depletion rate of media coverage $\lambda$, and it can be concluded that the bigger the $\lambda$, the larger the final densities of spreaders.

In this part, based on the theory of optimal control, combined with Pontryagin Maximum Principle, we respectively simulated the dynamic changes of four groups of people in different situations, such as Figures 5 and 6 . The two schemes are as follows: (1) without control: $u_{m}=0, u_{\delta}=0$, (2) with control: $u_{m} \neq 0, u_{\delta} \neq 0$. The parameters are set as shown in Table 1.

Figure 5 depicts the comparison of time evolution of spreaders and stiflers which applies both media coverage and emergency strategies by government to without control. As the diagram shows, in the presence of the optimal control variables $u_{m}$ and $u_{\delta}$, the peak value of spreaders is greatly reduced and the density is reduced to 0 faster than without control. Compared with the scheme without control, the time to reach the stability of the stiflers is shorter, and the final size is smaller. These phenomena show that the media coverage can make the ignorant know the rumor and reduce the infection rate of the ignorant. And the emergency strategies by government can effectively curb the spread of passion, so that the rumors will disappear as soon as possible.

Figure 6 shows the time evolution of the control variables $u_{m}$ and $u_{\delta}$. The maximum value at the beginning of the $u_{m}$ shows that the initial input of the media coverage is relatively large. As time evolves, the role of the media begins to play a scale effect, and the event is gradually clear and its cost decreases correspondingly. In the early stage of propagation, with the increase of spreader, the control variable $u_{\delta}$ gradually increased to the peak and then dropped to zero. This is because the government helps spreaders understand the truth by means of forcing segregation, holding press conferences, clarifying facts, and using other means, thus preventing spreaders from spreading rumors and transforming them into stiflers. As the density of the spreaders drops, the cost of the control variable is also corresponding reduction.

\section{Conclusion}

Based on the classical SIR model, an improved SIRM rumor spreading model is proposed. On the one hand, this paper 


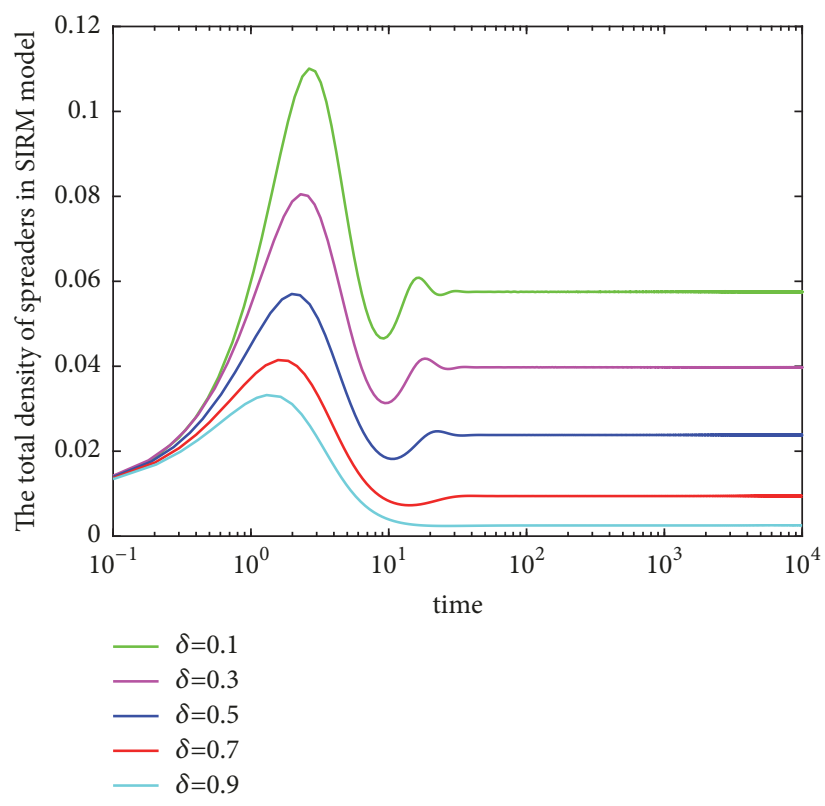

(a)

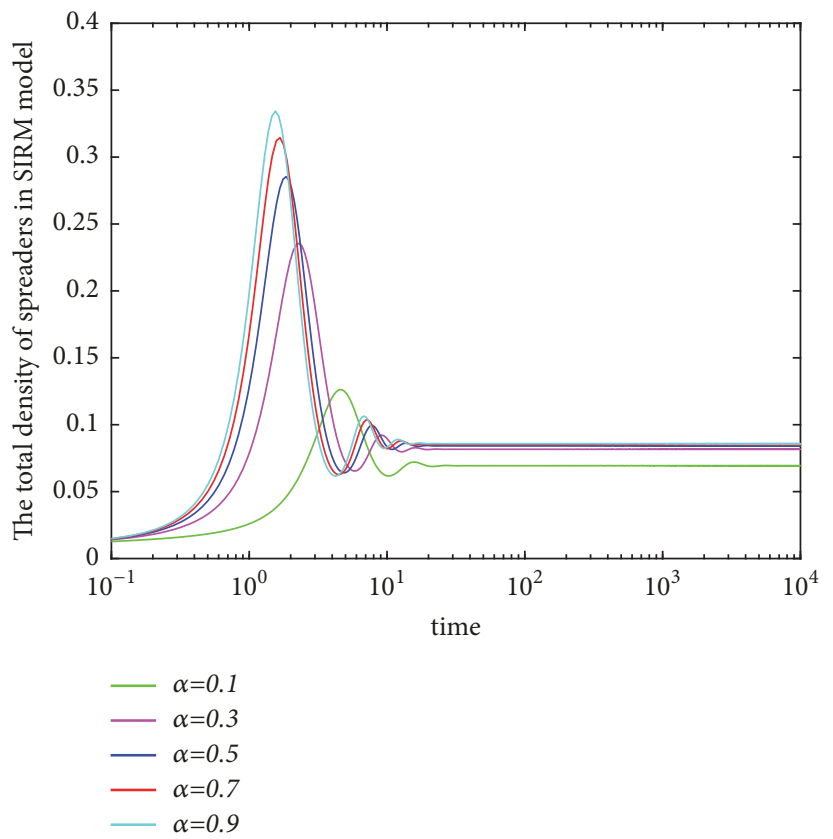

(c)

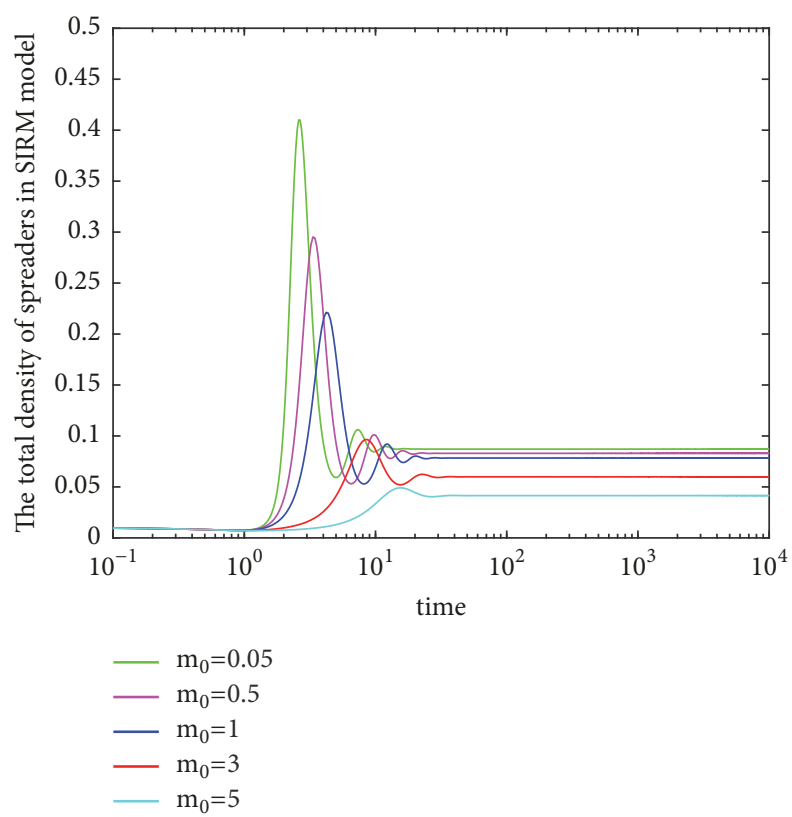

(b)
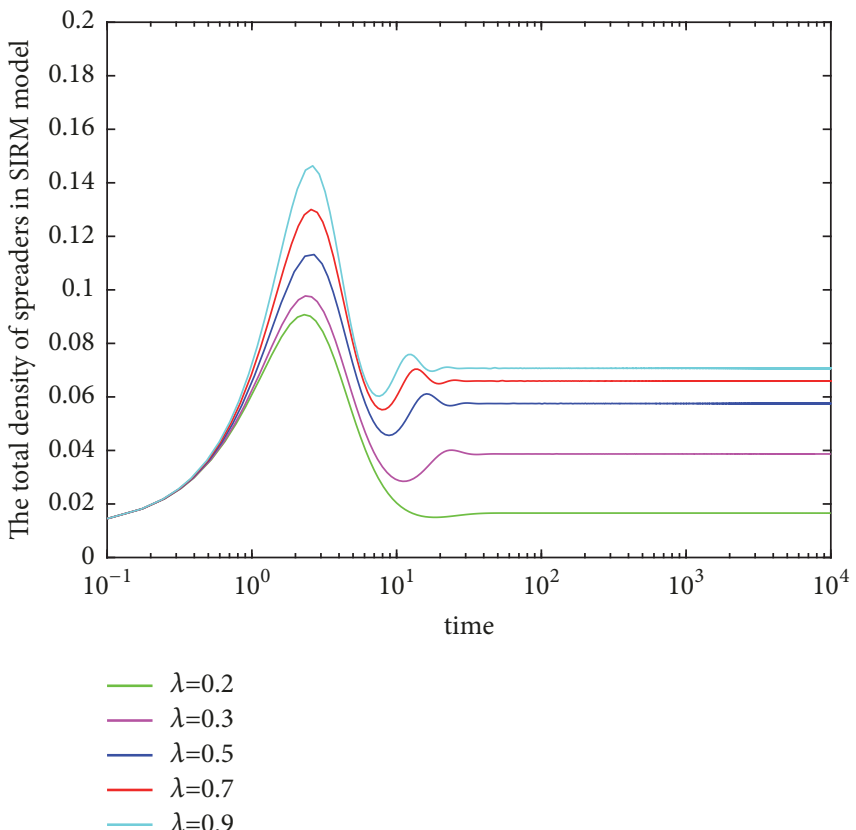

(d)

FIGURE 4: The impact of different values of $\delta, m_{0}, \alpha, \lambda$ on the total density of spreaders when $\Re_{0}>1$.

TABLE 1: Relevant parameter values.

\begin{tabular}{|c|c|c|c|c|c|c|c|c|c|c|c|c|c|}
\hline Parameters & $\Lambda$ & $\alpha$ & $\beta_{1}$ & $\beta_{2}$ & $\eta$ & $\varepsilon$ & $\delta$ & $\omega$ & $m_{0}$ & $\lambda$ & $A_{k}$ & $B_{k}$ & $C_{k}$ \\
\hline Values & 0.0001 & 0.5 & 0.133 & 0.133 & 0.001 & 0.000005 & 0.5 & 5 & 0.05 & 2 & 100 & 100 & 200 \\
\hline
\end{tabular}

introduces the media coverage into the rumor spreading model, and with the propaganda function of the media, the rumor can recognize the reality and thus manage the rumor from the source. On the other hand, the emergency strategies by government are also used to block the diffusion of spreaders. Then the dynamic equations are obtained and the threshold of rumor spreading is calculated. At the same time, for the purpose of maximizing the social utility and minimizing the cost of rumor governance, an optimal control problem has been formulated. The existence of an optimal control has been shown, and the corresponding optimality system has been derived. The proposed optimal control scheme can 


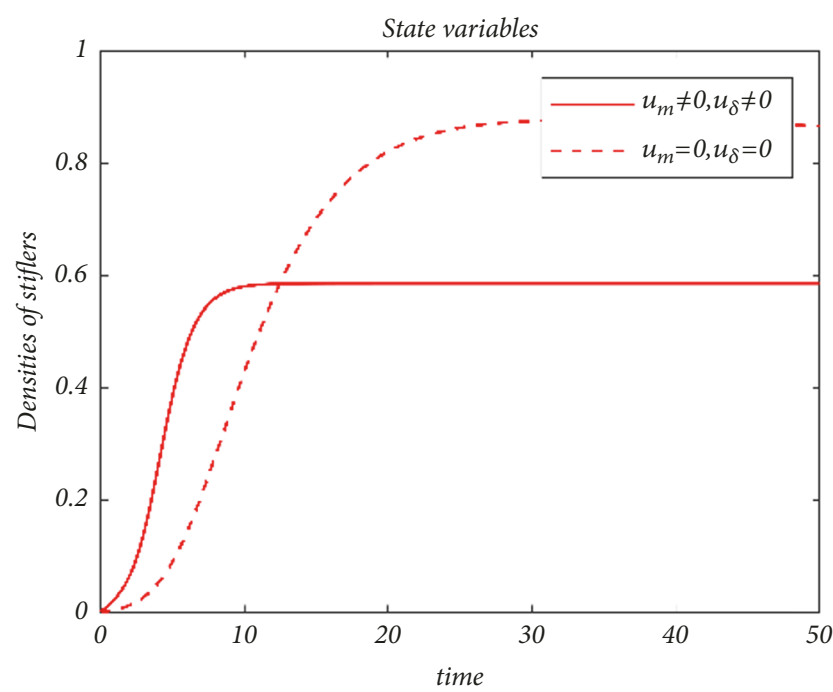

(a)

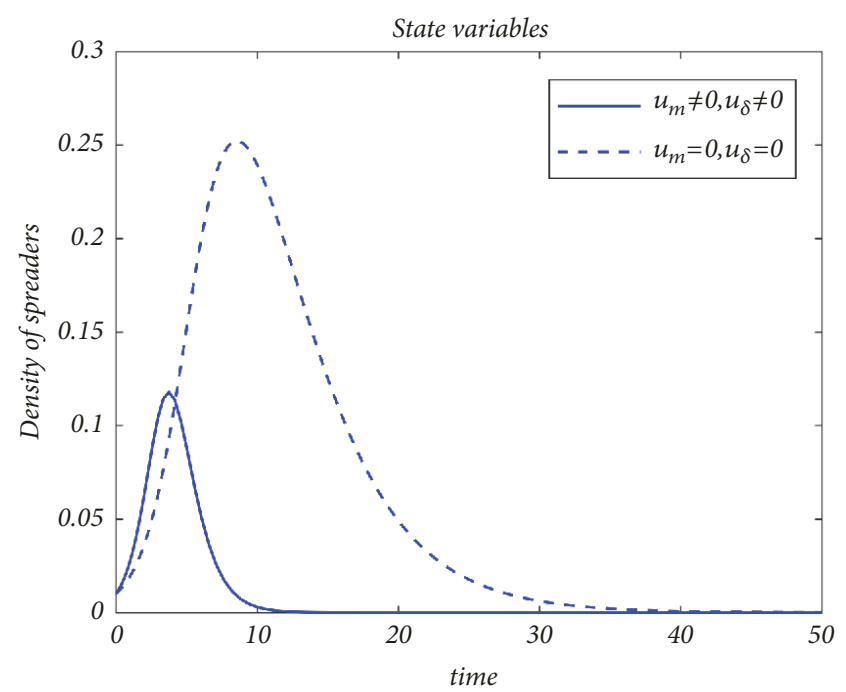

(b)

FIGURE 5: Evolution of densities of spreaders and stiflers with control or without control.

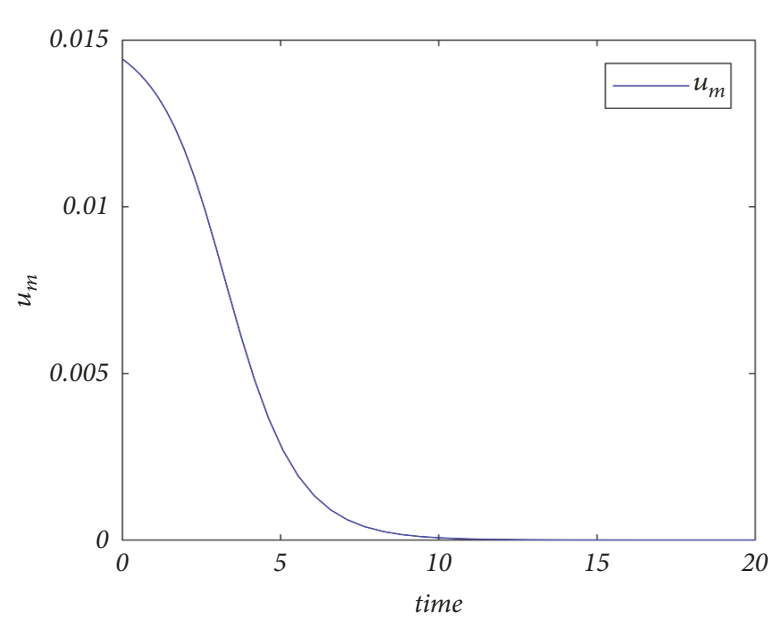

(a)

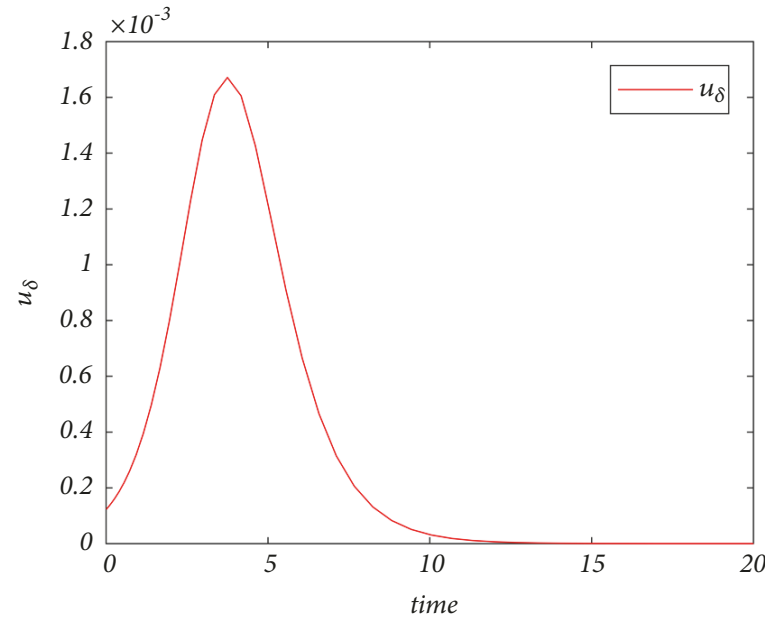

(b)

Figure 6: Time evolution of control variables ((a) for $u_{m}$, (b) for $u_{\delta}$ ).

achieve a low level of spreaders at a low cost. Finally, the theoretical analysis is verified by numerical simulation.

\section{Data Availability}

The data used to support the findings of this study are included within the article.

\section{Conflicts of Interest}

The authors declare that there are no conflicts of interest regarding the publication of this paper.

\section{Acknowledgments}

The work was partially supported by the National Natural Science Foundation of China (71774111).

\section{References}

[1] S. Galam, "Modelling rumors: the no plane Pentagon French hoax case," Physica A: Statistical Mechanics and its Applications, vol. 320, pp. 571-580, 2003.

[2] S. A. Thomas, "Lies, damn lies, and rumors: an analysis of collective efficacy, rumors, and fear in the wake of Katrina," Sociological Spectrum, vol. 27, no. 6, pp. 679-703, 2007.

[3] J.-H. Kim and G.-W. Bock, "A study on the factors affecting the behavior of spreading online rumors: Focusing on the rumor recipient's emotions," in Proceedings of the 15th Pacific Asia Conference on Information Systems: Quality Research in Pacific, PACIS 2011, Australia, July 2011.

[4] D. Liu and X. Chen, "Rumor propagation in online social networks like Twitter - A simulation study," in Proceedings of the 3rd International Conference on Multimedia Information Networking and Security, MINES 2011, pp. 278-282, China, November 2011. 
[5] A. Rapoport, "Spread of information through a population with socio-structural bias: I. Assumption of transitivity," Bulletin of Mathematical Biology, vol. 15, no. 4, pp. 523-533, 1953.

[6] W. Goffman and V. A. Newill, "Communication and epidemic processes," Nature, vol. 204, no. 4955, pp. 225-228, 1964.

[7] D. J. Daley and D. G. Kendall, "Stochastic rumours," Journal of the Institute of Mathematics and Its Applications, vol. 1, pp. 4255, 1965.

[8] K. Dietz, "Epidemics and rumours: A survey," Journal of the Royal Statistical Society. Series A. General, vol. 130, pp. 505-528, 1967.

[9] R. E. Dickinson and C. E. Pearce, "Rumours, epidemics, and processes of mass action: synthesis and analysis," Mathematical and Computer Modelling, vol. 38, no. 11-13, pp. 1157-1167, 2003.

[10] K. Thompson, R. Castro Estrada, and D. Daugherty, "A deterministic approach to the spread of rumors," Tech. Rep. BU1642-M, 2003.

[11] L. Huo, L. Wang, N. Song, C. Ma, and B. He, "Rumor spreading model considering the activity of spreaders in the homogeneous network," Physica A: Statistical Mechanics and its Applications, vol. 468, pp. 855-865, 2017.

[12] C. Liu, L.-x. Zhou, C.-j. Fan, L.-a. Huo, and Z.-w. Tian, "Activity of nodes reshapes the critical threshold of spreading dynamics in complex networks," Physica A: Statistical Mechanics and its Applications, vol. 432, pp. 269-278, 2015.

[13] C.-j. Fan, Y. Jin, L.-a. Huo, C. Liu, Y.-p. Yang, and Y.-q. Wang, "Effect of individual behavior on the interplay between awareness and disease spreading in multiplex networks," Physica A: Statistical Mechanics and its Applications, vol. 461, pp. 523-530, 2016.

[14] C. Fan, Y. Jin, L.-A. Huo, C. Liu, and Y. Yang, "Epidemic spreading of interacting diseases with activity of nodes reshapes the critical threshold," International Journal of Modern Physics C, vol. 28 , no. 1, 1750013, 12 pages, 2017.

[15] R. Ma, "Media, Crisis, and SARS: An Introduction," Asian Journal of Communication, vol. 15, no. 3, pp. 241-246, 2005.

[16] E. Klein, R. Laxminarayan, D. L. Smith, and C. A. Gilligan, "Economic incentives and mathematical models of disease," Environment and Development Economics, vol. 12, no. 5, pp. 707732, 2007.

[17] J. M. Tchuenche, N. Dube, C. P. Bhunu, R. J. Smith, and C. T. Bauch, "The impact of media coverage on the transmission dynamics of human influenza," BMC Public Health, vol. 11, no. 1, article S5, 2011.

[18] L. Zhao, Q. Wang, J. Cheng et al., “The impact of authorities' media and rumor dissemination on the evolution of emergency," Physica A: Statistical Mechanics and its Applications, vol. 391, no. 15, pp. 3978-3987, 2012.

[19] S. Collinson and J. M. Heffernan, "Modelling the effects of media during an influenza epidemic," BMC Public Health, vol. 14, pp. 376-386, 2014.

[20] J. M. Tchuenche and C. T. Bauch, "Dynamics of an Infectious Disease Where Media Coverage Influences Transmission," ISRN biomathematics, vol. 2012, Article ID 581274, 10 pages, 2012.

[21] Y. Luo and J. Ma, "The influence of positive news on rumor spreading in social networks with scale-free characteristics," International Journal of Modern Physics C, vol. 29, no. 09, p. 1850078, 2018.

[22] H.-F. Huo, P. Yang, and H. Xiang, "Stability and bifurcation for an SEIS epidemic model with the impact of media," Physica A: Statistical Mechanics and its Applications, vol. 490, pp. 702-720, 2018.
[23] H. Huo F and Y. Wang Y, "Impact of media coverage on the drinking dynamics in the scale-free network," SpringerPlus, vol. 5, no. 1, 2016.

[24] L.-A. Huo, P. Huang, and X. Fang, "An interplay model for authorities' actions and rumor spreading in emergency event," Physica A: Statistical Mechanics and its Applications, vol. 390, no. 20, pp. 3267-3274, 2011.

[25] Guanghua Chen, Huizhang Shen, Teng Ye, Guangming Chen, and Naphtali Kerr, "A Kinetic Model for the Spread of Rumor in Emergencies," Discrete Dynamics in Nature and Society, vol. 2013, Article ID 605854, 8 pages, 2013.

[26] D. Li and J. Ma, "How the government's punishment and individual's sensitivity affect the rumor spreading in online social networks," Physica A: Statistical Mechanics and its Applications, vol. 469, pp. 284-292, 2017.

[27] Xia-Xia Zhao and Jian-Zhong Wang, "Dynamical Behaviors of Rumor Spreading Model with Control Measures," Abstract and Applied Analysis, vol. 2014, Article ID 247359, 11 pages, 2014.

[28] L. Zhu and H. Zhao, "Dynamical behaviours and control measures of rumour-spreading model with consideration of network topology," International Journal of Systems Science, vol. 48, no. 10, pp. 2064-2078, 2017.

[29] L. Huo and C. Ma, "Optimal control of rumor spreading model with consideration of psychological factors and time delay," Discrete Dynamics in Nature and Society, Art. ID 9314907, 12 pages, 2018.

[30] K. Kandhway and J. Kuri, "How to run a campaign: optimal control of SIS and SIR information epidemics," Applied Mathematics and Computation, vol. 231, pp. 79-92, 2014.

[31] T. Lin, C. Fan, C. Liu et al., "Optimal control of a rumor propagation model with latent period in emergency event," Advances in Difference Equations, vol. 2015, no. 1, 2015.

[32] J. Xu, Y. Yu, C. Gao, and J. Sun, "Nonlinear analysis and optimal control of an improved SIR rumor spreading model," Journal of Communications, vol. 10, no. 8, pp. 638-646, 2015.

[33] Y. D. Jeong, K. S. Kim, and I. H. Jung, "Optimal control strategies depending on interest level for the spread of rumor," Discrete Dynamics in Nature and Society, Art. ID 9158014, 15 pages, 2018.

[34] P. van den Driessche and J. Watmough, "Reproduction numbers and sub-threshold endemic equilibria for compartmental models of disease transmission," Mathematical Biosciences, vol. 180, pp. 29-48, 2002.

[35] W. H. Fleming and R. W. Rishel, "Deterministic and Stochastic Optimal Control," Journal of Applied Mathematics and Mechanics, vol. 59, no. 9, 1975.

[36] D. L. Lukes, Differential equations: classical to controlled, Elsevier, 1982.

[37] S. M. Lenhart and J. T. Workman, Optimal Control Applied to Biological Models, CRC Press, 2007. 


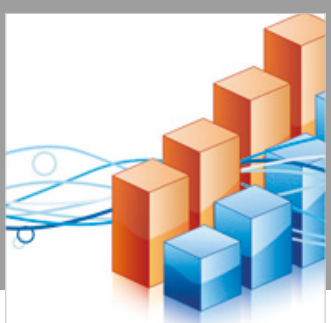

Advances in

Operations Research

\section{-n-m}
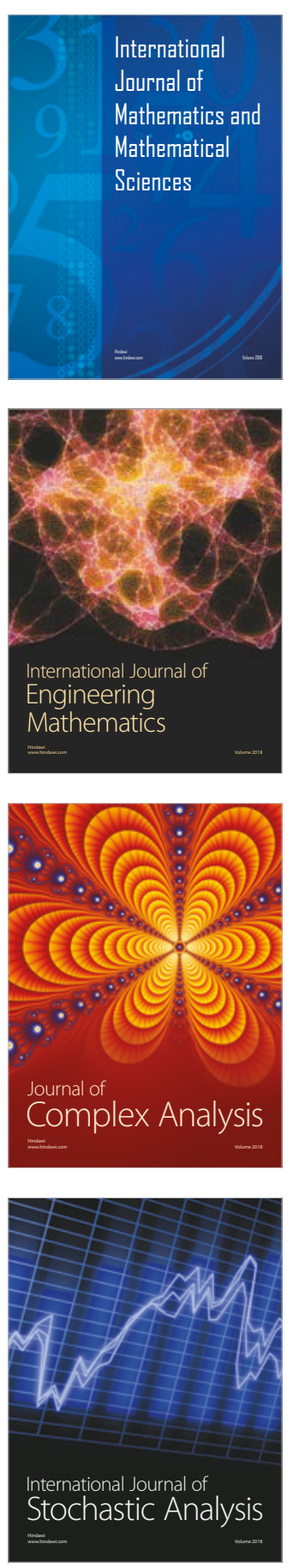
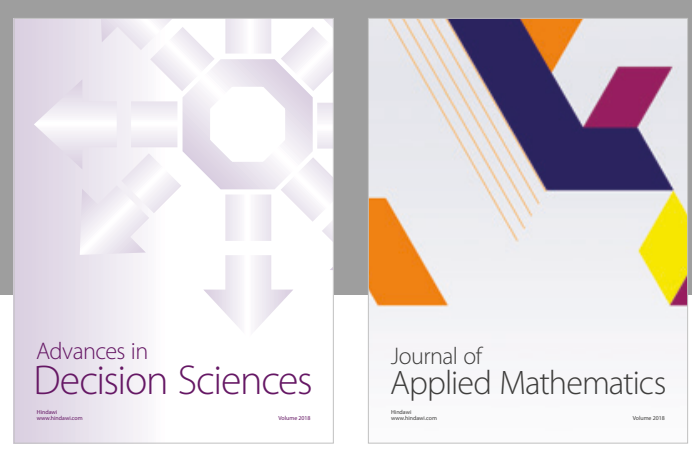

Journal of

Applied Mathematics
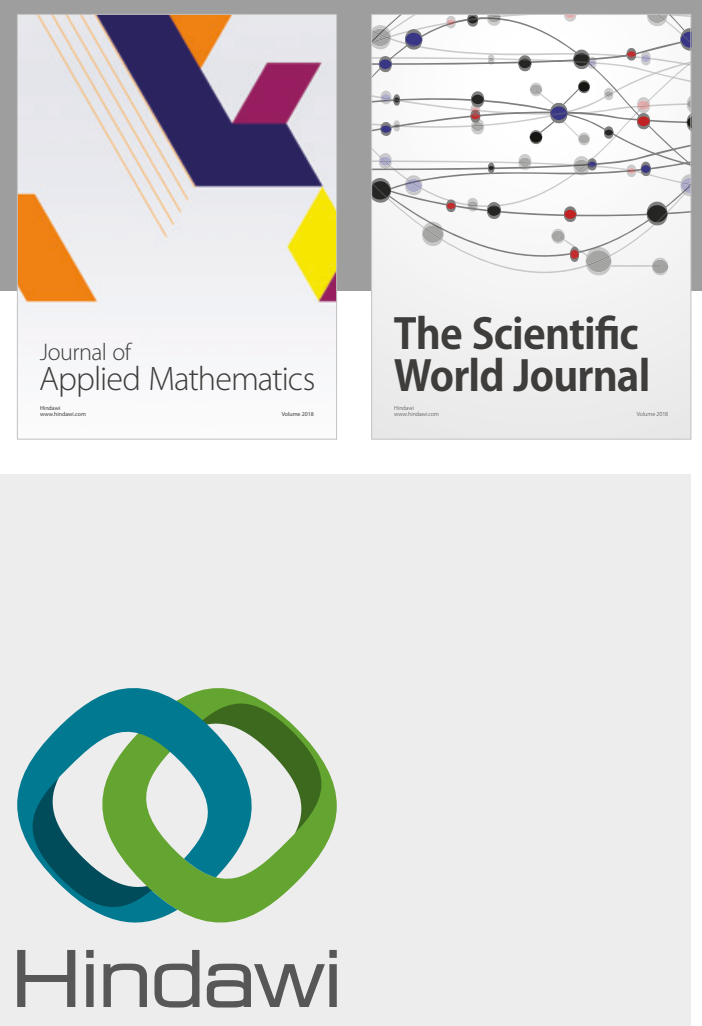

Submit your manuscripts at

www.hindawi.com

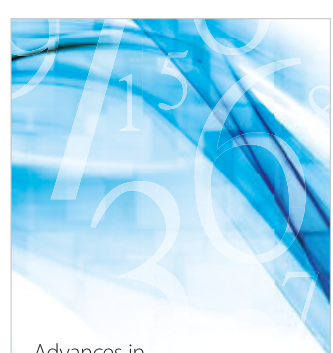

Advances in
Numerical Analysis
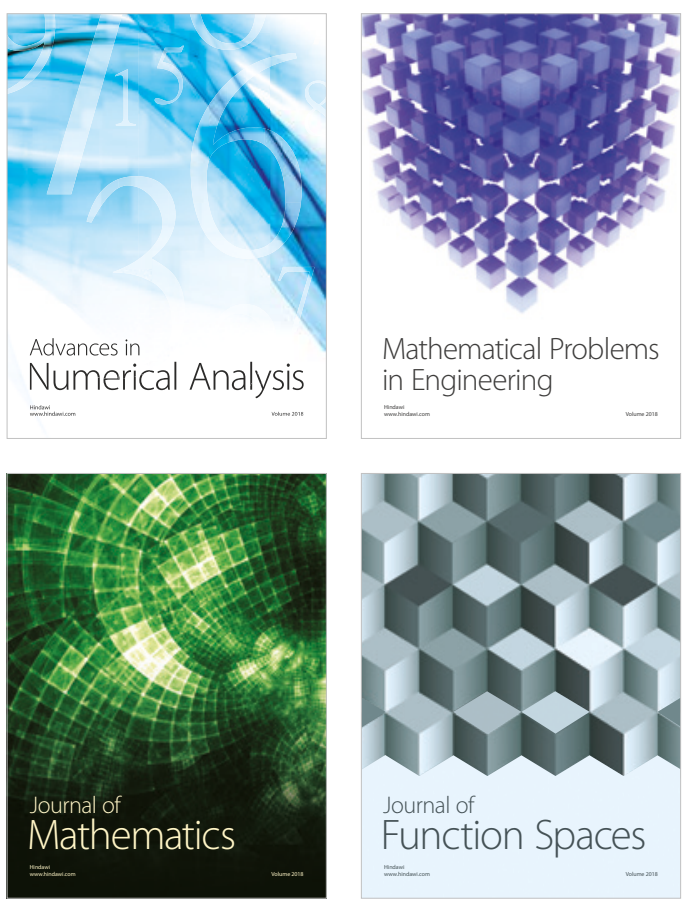

Mathematical Problems in Engineering

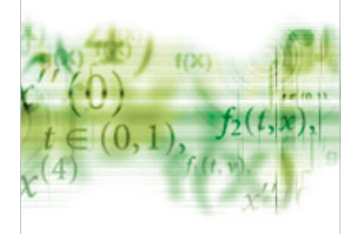

International Journal of

Differential Equations

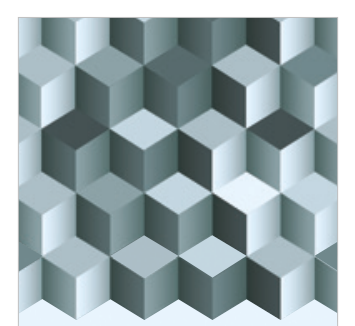

Journal of

Function Spaces

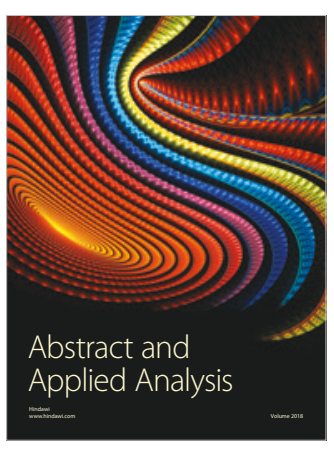

The Scientific

World Journal

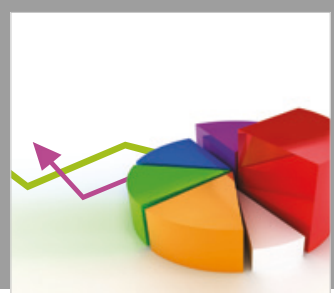

Journal of

Probability and Statistics
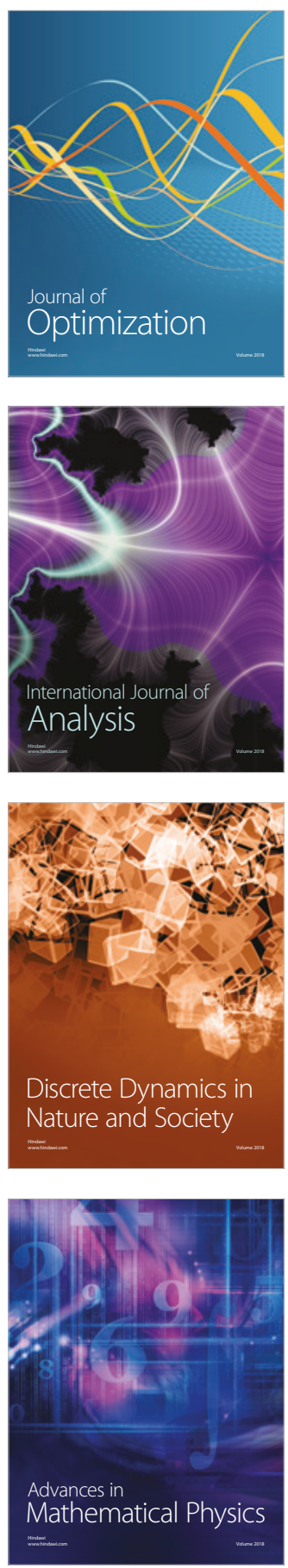